\title{
Competencias genéricas en la práctica profesional de la carrera Ingeniería Civil Eléctrica. El discurso entre la academia y la industria
}

\author{
Generic competences in the professional practice of the Electrical Engineering. \\ The discourse between academia and industry
}

\author{
Andrea Garrido Rivera ${ }^{1} \quad$ Fabricio Salgado Díaz ${ }^{2 *}$ \\ Carmen Paz Soto Caro ${ }^{3} \quad$ Paulette Blanc España ${ }^{4}$
}

Recibido 19 de marzo de 2019, aceptado 22 de octubre de 2020

Received: March 19, 2019 Accepted: Octubre 22, 2020

\begin{abstract}
RESUMEN
El propósito del estudio busca establecer la relación entre el discurso académico y el de los empleadores respecto a las competencias genéricas declaradas en el modelo educativo universitario, desarrolladas en la carrera de Ingeniería Civil Eléctrica, en la Universidad del Bío-Bío. Metodológicamente, corresponde a un estudio de tipo transaccional, que consideró una ruta investigativa situada en un paradigma cualitativo. Desde el punto de vista cualitativo, corresponde a estudio interpretativo, considerando un análisis de categoría de texto. Los principales resultados mostraron que: existe diferenciación entre ambos sectores (académico y profesional), respecto a la valoración de las competencias genéricas (conocidas como transversales), evidenciadas en los estudiantes de último año; además de mostrar que ambos sectores otorgan importancia a una formación de ingenieros integrales en sintonía a lo declarado por los estándares de referencia internacional, lo que implica igual desarrollo de competencias genéricas y específicas, aun cuando no mencionan formas de desarrollo en el proceso formativo de manera explícita quedando supeditadas a la voluntariedad de cada docente de la especialidad.
\end{abstract}

Palabras clave: Competencias, educación superior, ingeniería, prácticas profesionales.

\section{ABSTRACT}

The purpose of the study is to establish the relationship between academic discourse and that of employers regarding generic competences declared in the university educational model, developed in the Electrical Engineering at the Universidad del Bío-Bío. Methodologically, it is a transactional study, which considers a qualitative paradigm. From the qualitative point of view, an interpretive study is developed, considering an analysis of the text category. The main results showed differentiation between academic and professional sectors, regarding the valuation of generic competences (known as transversal competences), evidenced in seniors' students. Additionally, it is shown that both sectors attribute importance to the training of integral engineers according to international standards, which implies equal development of generic and specific competences, even when they do not mention forms of development in the training process in a straightforward way, being subject to the voluntariness of each teacher of the specialty.

Keywords: Competences, higher education, engineering, professional practices.

1 Universidad Católica de la Santísima Concepción. Facultad de Educación. Departamento de Currículum y Evaluación. Concepción, Chile.E-mail: andrea.garrido@ucsc.cl

2 Universidad del Bío-Bío. Facultad de Ingeniería. Dpto. Ingeniería Eléctrica y Electrónica. Concepción, Chile. E-mail: fsalgado@ubiobio.cl

3 Universidad Católica de la Santísima Concepción. Facultad de Educación. Concepción, Chile. E-mail: cpsoto@ucsc.cl

4 Universidad de Concepción. Concepción, Chile. E-mail: pblanc@udec.cl

* Autor de correspondencia: fsalgado@ubiobio.cl 


\section{INTRODUCCIÓN}

Durante el siglo XX la producción del conocimiento se maximizó a un nivel nunca antes visto, proceso que hoy sigue en alza, proyectando su desarrollo a un nivel prácticamente incuantificables. Este hecho, es el que ya desde el siglo pasado ha instalado la reflexión respecto a cuál sería la mejor forma de vincularnos hoy con el conocimiento. Cabe destacar, que gran parte de la producción de este conocimiento, se ha generado en las universidades. Lo anterior, hizo que, durante muchos años, la dinámica educativa que realizaban los centros de formación, estuviese dirigida a la preservación de la cultura, por medio de un paradigma transmisionista. Hoy se sabe, esto sería imposible, ya que se ha comprobado que gran parte de la información existente, por una parte, se renueva muy rápidamente y por otra, debido a que existe en gran volumen abordarlo bajo la misma figura es inviable. Como diría Alsina, et al., [1] hemos pasado de una relación de dominio del conocimiento a una de gestión de la información, lo que necesariamente, cambia la manera de entender los fines de formación y el desarrollo de la docencia, poniendo en tela de juicio, la forma en qué se entenderá el aprendizaje y también la evaluación. Este enfoque ha sido, el que, en conjunto con las perspectivas económicas, el fenómeno de la globalización, la influencia de las migraciones y la expansión tecnológica [2], el que medie el desarrollo de nuevas formas de entender el quehacer universitario y el sentido que tiene la formación de profesionales.

Según Villa y Poblete [3] es en la idea de contribuir al desarrollo de todas las facetas personales, sociales, culturales políticas y económicas, donde nace la necesidad de adaptación de las universidades a sus contextos nacionales e internacionales; además de la de prepararse para poder dar respuesta satisfactoria a los requerimientos de la sociedad y a los nuevos problemas, necesidades e intereses que están surgiendo.

Al respecto estos mismos autores señalan que posterior al acuerdo de Bolonia, se les solicita a las universidades que definan sus planes y programas, considerando la visión de diversos actores, vinculados con el desarrollo social y laboral incluyendo las competencias que hoy se consideran esenciales. Siendo justamente bajo esta lógica, que surge la vinculación con las necesidades expuestas por los graduados y/o profesionales respecto al desarrollo de dominios de la especialidad, y el desarrollo de competencias genéricas o transversales. Considerando como responsabilidad de la institución universitaria la formación también en este ámbito.

En ese sentido, cabe recordar algunos hitos que marcaron los procesos de reforma curricular en la Educación Superior a nivel global. Como se mencionó, el hito que marca la transición de un modelo tradicional a uno competencial, es el Proceso Europeo de Bolonia. Con el acuerdo suscrito, lo que se pretendía era conformar un sistema educativo europeo de calidad, que posibilitara a Europa fomentar el crecimiento económico, la competitividad internacional y su cohesión social a través de la educación y la formación de los ciudadanos a lo largo de la vida, además de su movilidad [4] .

A la implementación de Bolonia le siguió el Tuning Educational Structures in Europe (Proyecto Tuning) [5] y posteriormente Tuning América-Latina [6]. En Chile, la Reforma curricular, se inicia el año 2002, con los proyectos MECESUP 6 , y posterior a ello, a partir de la decisión del Consejo de Rectores, en el año 2003, de establecer un sistema de créditos académicos común a todas las universidades, materializándose el año 2006 como Sistema de Créditos Académicos Transferibles SCT-Chile [7], implementándose en prácticamente todas las universidades chilenas.

Es así, como diversos documentos oficiales, de relevancia internacional, han dado cuenta desde hace algunos años de manera explícita la importancia y necesidad de incluir en las declaraciones educativas y en el desempeño profesional los aspectos actitudinales y con posterioridad, las competencias transversales $[8,9]$.

En ese mismo sentido, se considera esencial el estar consciente de la naturaleza global de la profesión; de los desafíos tecnológicos, sociales, pero también de los ambientales, culturales y éticos, así como de las estrategias posibles para abordarlos [10]. Bajo esta misma lógica, es relevante, convirtiéndose incluso

\footnotetext{
5 Consejo de Coordinación Universitaria, Ministerio de Educación y Ciencia (2005). Espacio Europeo de Educación Superior (ESHE). Folleto de 6 p. Madrid. p. 2.

6 Proyecto de Mejoramiento de la Calidad de la Educación Superior.
} 
en una necesidad el vincular la academia con los demás actores del mundo social, ya sean públicos o privados, con un afán de realimentación de sus requerimientos desde una perspectiva bidireccional, tal como los señala por ejemplo la experiencia relatada por Falcone, Vázquez, García y López-Martin [11].

En ese mismo contexto, el artículo presenta los resultados del trabajo investigativo que da cuenta de la forma en que la academia y el sector industrial valoran el desarrollo de las competencias genéricas declaradas por el modelo educativo de la Universidad del Bío-Bío. En ese sentido, se presenta primero, la referencia teórica, luego el diseño metodológico, seguido del cual se dan a conocer los principales resultados. Por último, se enuncian las conclusiones, que dan respuesta a los objetivos propuestos.

\section{OBJETIVOS Y SUPUESTO DE INVESTIGACIÓN}

El objetivo general de este estudio es: Analizar la relación entre el discurso académico y de la industria respecto al abordaje de las competencias genéricas declaradas en el modelo educativo de la Universidad del Bío-Bío, en los procesos de práctica profesional de la carrera de Ingeniería Civil Eléctrica.

Del que se desprenden los siguientes objetivos específicos:

1. Identificar en el discurso académico la presencia de las competencias genérica declaradas por la universidad en el proceso de práctica profesional de la carrera de Ingeniería Civil Eléctrica.

2. Describir desde la visión del supervisor de práctica de la industria sobre el desempeño basado en competencias genéricas de los estudiantes en práctica profesional en la carrera de Ingeniería Civil Eléctrica. Y,

3. Contrastar el discurso de los académicos e industrial, las visiones del mundo académico y de las empresas respecto a las competencias genéricas en la carrera de Ingeniería Civil Eléctrica.

También durante el estudio se dará respuesta al siguiente supuesto de investigación:

- Los discursos de los académicos y de la industria difieren en la forma conceptualizar las competencias genéricas, así como también en la forma en que las valoran el desarrollan de estas en los estudiantes.

\section{MARCO DE REFERENCIA}

\section{El contexto de las competencias genéricas}

Como ya se ha mencionado las instituciones educativas no pueden ignorar los cambios sociales y tecnológicos que se están produciendo, pero es importante considerar este aspecto, no sólo porque la Universidad debe preparar individuos que sepan desarrollarse en el campo profesional y personal, sino también porque estos jóvenes están creciendo en ese contexto de fluctuaciones continuas, lo que significa maneras diferentes de pensar a las de la generación anterior.

Si partimos de esta premisa podemos mencionar que los énfasis presentados responderían a una forma distinta de entender el proceso de formación, lo que implica necesariamente una gestión docente distinta, así como también una forma distinta de relacionarnos con el conocimiento y de entender la Educación Superior. Bajo esta misma lógica es que surgen los denominados enfoques por competencias definidos hace un par de décadas ampliamente [3, $6,12,13]$, dando lugar a una variedad de enfoques, algunos de ellos, incluso muy contradictorios entre sí.

En cuanto al término competente, se refiere a que la persona practica y desarrolla diferentes capacidades y aptitudes, con el objetivo de dar respuesta a una situación problemática determinada [1]. De acuerdo a la literatura en la acción de asumir una competencia se evidencian aspectos tales como: la exploración, el conocimiento, la comprensión, la aplicación, el análisis, la síntesis y la evaluación de la gestión del proyecto o la actividad académica que un sujeto debe trabajar ya sea tanto individual como colectivamente. En definitiva, el asumir esta postura se relaciona a la consideración de la múltiple dimensionalidad del aprendizaje con énfasis en la formación integral.

Por otro lado, el concepto de competencia genérica o transversal se invoca únicamente para designar una competencia que se necesita desde un punto de vista extenso en un rango de diferentes e importantes demandas cotidianas, profesionales y para la vida social. Las competencias se necesitan para lograr diferentes e importantes metas y resolver múltiples problemas en variados contextos desde lo familiar y cotidiano hasta lo complejo y desconocido [14].

Así Poblete y otros [15] recomienda seleccionar las competencias acordes con determinados criterios, 
destacando entre ellos los siguientes a) Las competencias genéricas son consistentes con los principios de los derechos humanos y los valores democráticos; b) Las competencias transversales desarrollan la capacidad individual para una buena y exitosa vida y c) las competencias genéricas no son incompatibles con la diversidad individual y social.

Se añaden aquí también algunas características a contemplar en la definición y consideración de las competencias genéricas, tales como que: Las competencias genéricas son integradoras de las capacidades humanas, las competencias desarrollan la autonomía de las personas y que además desarrollan la significatividad del aprendizaje.

Al respecto, Orsini [16], menciona la relación entre lo genérico y la transversalidad, plantea que el concepto de transversalidad es aquel contenido, tema, objetivo o competencia que atraviesa todo el proceso de enseñanza aprendizaje porque busca establecer conexiones entre lo disciplinario y lo formativo para lograr aprendizaje. Al presentar este objetivo, la transversalidad interpela no sólo al currículum oficial, sino también a la cultura escolar y los actores que forman parte de ella.

La idea es proponer una mirada sistémica, que pueda apoyar la equidad de la educación. Por tanto, la transversalidad es un enfoque dirigido al mejoramiento de la calidad educativa, buscando evitar la fragmentación de las áreas del conocimiento, a partir del desarrollo de una visión holística, que promueva además la aprehensión de valores, la formación de actitudes, la expresión de sentimientos, maneras de entender el mundo y a las relaciones sociales en un contexto específico.

Pese a que todas ellas, dan cuenta de la necesidad de abordar la temática, en cada uno de los niveles educativos (básicos, secundarios y universitarios), al parecer durante todos estos años ha habido falta de acuerdo ante como hacerlo, y más aún respecto de su significado [17, 18], tampoco respecto de cuáles serían, cómo deben ser trabajadas, y por último, como deberían ser evaluadas [3, 19, 20, 21, 22, 23, 24].

\section{Competencias genéricas en ingeniería}

Desde la National Academy of Engineering de EE.UU. (2004) y Royal Academy of Engineering del Reino Unido [25], se reconocen como aspectos deseables el que los ingenieros puedan desarrollar los siguientes atributos: Competencias sociales y personales para trabajar en equipos diversos, razonar en forma crítica y creativa, ejercer liderazgo; realizar iniciativas emprendedoras; comunicarse efectivamente en lengua materna e inglés; auto gestionarse en contextos exigentes, complejos e incertidumbres.

Por su parte, la visión de la American Society of Engineering Education (1994) [26] señala dentro de los atributos requeridos que demuestra una compresión de las perspectivas políticas, sociales y económicas; demuestra una compresión de las tecnologías de la información, las competencias digitales y la alfabetización e información; demuestra una compresión de las normas éticas y de negocios y aplica tales nomas de manera efectiva en un contexto dado (organización, sector y país); se comunica de manera efectiva en una variedad de formas, métodos y de medios de comunicación (escrita, verbal/oral, gráfica, auditiva y electrónica); posee la habilidad de pensar crítica y creativamente; posee habilidad de pensar individual y cooperativamente; Funciona efectivamente en un equipo (entiende las metas del equipo, contribuye efectivamente al trabajo en equipo, apoya las decisiones del equipo, respeta a los miembros del equipo, etc.); Mantiene una imagen personal positiva y tiene autoconfianza positiva; adopta un compromiso con los principios y estándares de calidad y con el mejoramiento continuo; adopta perspectivas interdisciplinarias y multidisciplinarias; aplica juicio personal y profesional para tomar decisiones efectivas y gestionar los riesgos; apoya otros para alcanzar las metas y realizar tareas; muestra iniciativa y demuestra voluntad en emprender.

Así también los criterios americanos, $\mathrm{ABET}^{7}$ [27] para la certificación de calidad de los programas de ingeniería plantean ciertos descriptores que se vinculan directamente con los aspectos genéricos, donde 5 de los 7 resultados correspondientes al criterio 3 de ABET, evidencian elementos asociados a la consideración aspectos transversales: 1 . La capacidad de aplicar el proceso de diseño de ingeniería para producir soluciones que satisfagan

\footnotetext{
7 Desde sus inicios el término ABET era el acrónimo de Accreditation Board for Engineering and Technology, pero desde el año 2005 se usa la sigla para hacer referencia a la organización.
} 
necesidades específicas teniendo en cuenta la salud y la seguridad públicas, y factores globales, culturales, sociales, ambientales, económicos y de otro tipo, según corresponda a la disciplina; 2. Capacidad para comunicarse de manera efectiva con una variedad de audiencias; 3 . La capacidad de reconocer responsabilidades éticas y profesionales en situaciones de ingeniería y emitir juicios informados, que deben considerar el impacto de las soluciones de ingeniería en contextos globales, económicos, ambientales y sociales; 4 . La capacidad de reconocer la necesidad constante de adquirir nuevos conocimientos, elegir estrategias de aprendizaje apropiadas y aplicar este conocimiento. 5. La capacidad de funcionar eficazmente como miembro o líder de un equipo que establece objetivos, planifica tareas, cumple plazos y crea un entorno colaborativo e inclusivo.

Como se puede ver, todos los marcos de referencia de la ingeniería vinculan directamente los aspectos técnicos disciplinares con competencias genéricas haciendo visible la multidimensionalidad tal como enfatiza Unesco en la siguiente cita:

La ingeniería debe guiar los procesos económicos, sociales y humanos y sostener el conocimiento de las sociedades, así también debe ser un aporte a la innovación y al desarrollo de las sociedades globales [28].

Se puede decir por tanto, que entre las tendencias internacionales en la formación de los ingenieros se destacan: la formación virtual y los nuevos ambientes de aprendizaje, la incorporación de las Tecnologías de la Información y las Comunicaciones (TIC) en el aula de clase, la ética asociada a las decisiones de ingeniería, el crecimiento acelerado de los cursos y denominaciones de ingeniería, el aseguramiento de la calidad de los programas académicos, y el aporte social de la ingeniería, entre otras. Los nuevos profesionales de ingeniería deben formarse bajo una premisa elemental: el ingeniero es un ser social de acción global [29].

\section{Medición y evaluación de las competencias genéricas}

En ese mismo contexto, orientadores son los trabajos de Eizaguirre, Altuna, Pikabea, Marko \& Pérez [30], quienes han desarrollado estudios respecto a un sistema de medición de las competencias en una carrera universitaria, encontrando diversos hallazgos los que pueden ser perfectamente transferibles a todos los contextos y niveles formativos. Entre los principales resultados se encontró que: a) Se observa cierta ausencia de conexión entre las diversas asignaturas y la gran diferencia de trabajo entre unas competencias y otras. b) Se aprecia la necesidad de acotar y clarificar las distintas definiciones de las competencias básicas y de las competencias transversales. c) A su vez, desde el principio del grado y queriendo buscar una mejor comprensión, se debieran explicar todas las competencias a desarrollar durante el grado al alumnado, mencionando la importancia que tienen para su desarrollo personal y profesional. d) Se debería asociar más aún, si cabe, las competencias que se trabajan en el practicum. Éste se considera un espacio idóneo para trabajar y evaluar la mayoría de las Competencias transversales. y e) Se añade un componente externo a la universidad donde se incluye una evaluación externa por parte del "instructor" o tutor de prácticas, lo que ayudaría a completar la valoración final o global de cada estudiante, en relación a estos aspectos.

Así mismo el autor, también propone algunos facilitadores entre los que destacan: el desarrollo de una evaluación continua, con instrumentos y claros objetivos a alcanzar, con criterios e indicadores concretos, para facilitar una valoración objetiva, a la vez sencilla. A su vez, estos autores proponen trabajar las competencias, desde la auto orientación. De manera que el estudiantado se involucre reflexivamente desde su vivencia, genere autoconocimiento e intenten mejorar los aspectos considerados aun en desarrollo.

Tal como advierten Medina, Sánchez y Pérez [31] y Culver y Cols [32], la lógica de las competencias requiere del diseño de pruebas que las consideren en su globalidad y complejidad, a la vez que evidencien el avance del sujeto en tal dominio. Le Boterf, propone modificar las prácticas de la evaluación y validación de las competencias, así como las formas de organización del trabajo y la estimación de ellas, en sus palabras: "Se requiere una evaluación colegiada, así como la implicación de los estudiantes, autoevaluación, informes (tutores), coevaluación y el dominio y puesta en práctica de las competencias profesionales estimadas por los empleadores" [33]. 
Por otra parte, se hace énfasis, en la coherencia que debe existir entre el modelo de formación y el de la evaluación de las competencias, además de que este ha de ser flexible, comprensivo e integrado [34]. Así también, la evaluación de las competencias debe considerar criterios de calidad y dominio de tales competencias, con claro conocimiento y referencia a los/las estudiantes y al equipo de docentes. La evaluación de las competencias debe considerar, un contexto de conocimiento reflexivo y de profundo avance, mediante la autoobservación, el análisis de las necesidades y la valoración y seguimiento de las metas a alcanzar.

En ese mismo plano se señala que la evaluación de actitudes y valores, ha de estar necesariamente integrada tanto en el conjunto de la labor educativa llevada a cabo, como en la apreciación global del progreso educativo del estudiante [35]. La distinción tripartita de contenidos (conceptos, procedimientos y actitudes), aun cuando señale formas diferenciales de enseñanza y evaluación, no debe significar que cada tipo de contenidos pueda tener una calificación diferenciada.

Por último, es importante destacar el planteamiento de Bolívar [36, 37], quien señala que la evaluación del ámbito de los transversales es uno de los aspectos más complejo, dependiendo de cómo se hayan incorporado en el Proyecto de Centro y del grado de consenso alcanzado en la comunidad colegiada, así como también por la consideración del triple componente (cognitivo, afectivo y comportamental) lo que hace que su evaluación tenga que ser integradora. Determinando indicadores, criterios, o pautas de observación, que guíen el propio proceso de observación, lo que hoy se observa aún como un desafío para la academia con escasa formación pedagógica.

\section{METODOLOGÍA}

Desde la investigación se asume la idea de que la realidad social por su naturaleza no es estática, ni objetiva, y que, muy por el contrario, esta se construye, existiendo una visión comprensiva de la misma, donde la subjetividad se hace presente y se valida. En ese contexto, y dadas las particulares teórico-empíricas, se trabaja desde una concepción epistémica interpretativa, con un enfoque metodológico cualitativo.
La caracterización tipológica de la unidad de estudio, responde a un muestreo opinático, intencionado, no probabilístico, y en ambos casos la muestra es el universo. Cabe destacar, que como todo estudio de naturaleza cualitativa, lo que se busca es profundizar a partir de las particularidades que ofrece el caso la realidad académica en un contexto determinado y no generalizar resultados. El proceso de recolección de datos se consideró el desarrollo de entrevistas semiestructuradas, y cuestionarios de consulta con ítem predefinidos y otros abiertos, los que fueron analizados para el caso de lo cualitativo, considerando el análisis de contenido de texto, bajo el criterio de interpretación de categorías propuesto por Ruíz Olabuénaga [38] y Flores [39].

Los aspectos personales y contextuales de los tres casos seleccionados son presentados en la Tabla 1.

\section{RESULTADOS}

\section{Análisis del discurso académico y empresarial}

El análisis del discurso de los académicos entrevistados se realiza considerando el modelo de categorías levantadas por la investigación. Se consideraron para este apartado categorías especiales, es decir propias del ámbito de estudio y emergentes. A saber: Presencia de las competencias genéricas, la que a su vez incluye como subcategoría modelo y plan de estudios, programa de asignatura, metodología y evaluación; importancia de las competencias genéricas; pertinencia de la competencia; y proceso reflexivo como una categoría emergente.

Por otro lado, el análisis del discurso de la empresa se hará considerando el mismo modelo categorial anteriormente descrito. Para ello, se contó con la opinión de 27 supervisores, identificados como $\mathrm{S} 1, \mathrm{~S} 2$, S3 sucesivamente; correspondientes a las empresas anteriormente mencionadas, conformando el universo total.

Se han utilizado como insumos, la totalidad de los informes de práctica para determinar la visión de la empresa respecto al desempeño de la competencia genérica de los/las estudiantes. Sin embargo, cabe destacar como una observación, que, en el apartado de comentarios incluido en la Pauta de Evaluación de la Práctica; no todos los supervisores expresaron su opinión dejando seis de ellos en blanco. Lo que reduce el tamaño de la data para el proceso de análisis. 
Tabla 1. Descripción de Informantes claves.

\begin{tabular}{|c|c|c|c|}
\hline Característica & $\begin{array}{l}\text { Informante } 1 \\
\text { Docente } 1\end{array}$ & $\begin{array}{c}\text { Informante } 2 \\
\text { Docente } 2\end{array}$ & $\begin{array}{c}\text { Informante } 3 \\
\text { Docente } 3\end{array}$ \\
\hline Edad & 41 & 72 & 41 \\
\hline Género & Masculino & Masculino & Masculino \\
\hline Título Profesional & Ingeniero Civil Eléctrico. & Ingeniero Civil Industrial, con mención en eléctrica. & Ingeniero Civil Electrónico. \\
\hline Formación permanente & Magister en Ciencias de la Ingeniería eléctrica. & Doctor en Educación. & Doctor en Ciencias de la Ingeniería. \\
\hline Años de experiencia & 12 años & 50 años & 10 años \\
\hline Años de experiencia en la UBB & 10 años & 49 años & 3 meses \\
\hline $\begin{array}{l}\text { Vinculación con el trabajo } \\
\text { de prácticas }\end{array}$ & Evaluador de prácticas. & $\begin{array}{l}\text { Revisor de informes, no ha realizado } \\
\text { supervisiones. }\end{array}$ & Evaluador de prácticas. \\
\hline
\end{tabular}

Fuente: Elaboración propia.

Tabla 2. Matriz de análisis cualitativo sobre competencias genéricas.

\begin{tabular}{|c|c|c|}
\hline Modelo y plan de estudio & Evidencia docente & Evidencia empresa \\
\hline $\begin{array}{l}\text { Se reconoce la presencia de aspectos transversales o actitudinales en } \\
\text { el modelo, pero se evidencian impresiones al momento de declarar } \\
\text { las competencias que incluye el modelo. En su relato mencionan } \\
\text { comunicación efectiva, uso de tecnología, capacidad de empatía, } \\
\text { comprensión del medio, habilidades comunicativas, que, aunque se } \\
\text { relacionan no son todas competencias genéricas declaradas por la } \\
\text { Universidad del Bío-Bío. Ahora bien, al momento de recordárselas } \\
\text { los tres docentes, asienten que las mismas estarían declaradas } \\
\text { contenidas en el plan de estudios de su carrera. } \\
\text { El académico } 1 \text { (A1) y el académico } 3 \text { (A3), consideran que las } \\
\text { competencias genéricas se deben trabajar mayoritariamente en los } \\
\text { cursos de formación integral y no en los cursos de especialidad. } \\
\text { Uno de los docentes menciona que todas las competencias declaradas } \\
\text { se trabajan en el plan de estudios existiendo diversas instancias para } \\
\text { su desarrollo. } \\
\text { Se refuerza la idea de la separación de lo disciplinar o la especialidad } \\
\text { con el desarrollo de competencias genéricas, dejando éstas últimas } \\
\text { cubiertas sólo por el área curricular de formación integral. }\end{array}$ & $\begin{array}{l}\text { "No es preciso en cada ramo de formación dura" (A1). } \\
\text { "... sino que las asignaturas están más orientadas a } \\
\text { lo principal o sea la competencia específica..." (A3). } \\
\text { "Se trabajan todas, porque el alumno tiene que pasar por } \\
\text { todas, tiene que cubrir las competencias genéricas a lo } \\
\text { largo del plan de estudio, y en total el alumno toma dos } \\
\text { de cada una y al final cuando se hace el conteo termina } \\
\text { con tres de una y dos de cada una, por la cantidad de } \\
\text { formaciones integrales que tenemos en el plan, las } \\
\text { cubre todas" (A3). } \\
\text { "El plan de estudio permite que el alumno tenga esas } \\
\text { competencias, pero para mejorarlas sería bueno que se le } \\
\text { diera más importancia dentro de la especialidad" (A3). }\end{array}$ & $\begin{array}{l}\text { "Facilidad de aprendizaje en las } \\
\text { distintas actividades encomen- } \\
\text { dadas" (S3). } \\
\text { "Buena capacidad de aprendizaje" } \\
\text { (S4). } \\
\text { "Gran facilidad de aprendizaje" } \\
\text { (S6 y S9). } \\
\text { "Alumno con mucha capacidad de } \\
\text { aprender" (S19). } \\
\text { "Con voluntad de aprendizaje" (S23). } \\
\text { "Debe trabajar habilidades blandas } \\
\text { de redacción de informes técnicos y } \\
\text { de expresión oral en reuniones"(S1). } \\
\text { "Falta desarrollar el aspecto comuni- } \\
\text { cativo" (S4). } \\
\text { "Deben mejorar el ámbito comuni- } \\
\text { cacional" (S22). }\end{array}$ \\
\hline Importancia de las competencias genéricas & Evidencia docente & Evidencia empresa \\
\hline $\begin{array}{l}\text { Todos los académicos le dan importancia a las competencias } \\
\text { genéricas, para ellos son fundamentales en la formación de un } \\
\text { ingeniero. Dentro de las competencias que destacan están: el } \\
\text { autoaprendizaje y la comunicación, la responsabilidad social (A1), } \\
\text { y al liderazgo y trabajo colaborativo (A3). Por su parte, los docentes } \\
\text { (A2) y (A3), declaran que es importante que desde el principio se } \\
\text { desarrollen las competencias genéricas. El docente (A3) reconoce } \\
\text { que existe un status inferior de las competencias genéricas respecto } \\
\text { de las específicas. Sin embargo, también reconoce que ambas son } \\
\text { importantes para el itinerario formativo de un ingeniero, se menciona } \\
\text { que, si bien se debiera apuntar hacia la integración de ambas, este } \\
\text { ejercicio no debiera suponer el debilitamiento de una competencia } \\
\text { genérica o específica. } \\
\text { Se argumenta, además que es el medio nacional e internacional, } \\
\text { el que enfatiza en el desarrollo de éstas competencias. Además, } \\
\text { se menciona como importante la necesidad de coordinación de } \\
\text { todas las unidades para la adecuada implementación de un modelo } \\
\text { adecuado de integración de competencias. } \\
\text { Los supervisores de la empresa consideran como importante los } \\
\text { siguientes aspectos: Capacidad de aprendizaje en los estudiantes, } \\
\text { las habilidades comunicativas, la disposición a creación de redes y } \\
\text { la disposición para realizar las tareas encomendadas. }\end{array}$ & $\begin{array}{l}\text { "El autoaprendizaje es importante porque cuando salgan } \\
\text { de acá deberán resolver problemas, y la resolución de } \\
\text { problemas es parte de los ingenieros civiles" (A1). } \\
\text { "Probablemente lo que se enseñe en la Universidad queda } \\
\text { obsoleto en ocho meses, después de eso debe seguir } \\
\text { aprendiendo y buscando sus propias herramientas"(A1). } \\
\text { "...incluso más, uno teniendo una carrera profesional } \\
\text { se dice que recién empieza a estudiar" (A2). } \\
\text { "Las principales el trabajo colaborativo, y también la } \\
\text { capacidad de comunicación a parte que el liderazgo } \\
\text { en lo que son las capacidades profesionales se ha } \\
\text { mencionado bastante en los supervisores, la capacidad } \\
\text { de liderazgo" (A3). } \\
\text { "Otra competencia genérica importante, es la capacidad de } \\
\text { emprendimiento, que es parte de una de las competencias } \\
\text { genéricas del modelo, y que lo menciona (A1). } \\
\text { "Donde muchos de ellos van a tener que crear empleos, } \\
\text { el primer empleo es propio" (A1). }\end{array}$ & $\begin{array}{l}\text { "El estudiante presenta una buena } \\
\text { disposición para realizar las tareas } \\
\text { encomendadas" (S14 y S15). } \\
\text { "Buena disposición para asumir } \\
\text { nuevas tareas" (S 14). } \\
\text { "Muy buena disposición para asu- } \\
\text { mir tareas encomendadas" (S9, } \\
\text { S10 y S15). } \\
\text { "Debe mejorar el ámbito comu- } \\
\text { nicacional para generar redes que } \\
\text { faciliten el desarrollo de su trabajo" } \\
\text { (S22). }\end{array}$ \\
\hline
\end{tabular}




\begin{tabular}{|c|c|c|}
\hline Pertinencia contexto profesional & Evidencia docente & Evidencia empresa \\
\hline $\begin{array}{l}\text { Dos de los tres entrevistados considera las competencias genéricas } \\
\text { pertinentes al contexto de desarrollo profesional, vinculándolas } \\
\text { directamente con las competencias específicas, como liderazgo, } \\
\text { comunicación, autoaprendizaje y resolución de problemas. } \\
\text { Es la industria la que ha creado la necesidad de abordar las } \\
\text { competencias blandas en la formación profesional y que demanda otro } \\
\text { tipo de competencias, no sólo las competencias técnicas o específicas. } \\
\text { El docente } 3 \text { (A3) se muestra receptivo a los aspectos declarados } \\
\text { en este ámbito en el contexto internacional, donde se refuerza la } \\
\text { idea de formación integrada, es decir sinergia entre competencias } \\
\text { específicas y competencia genéricas. } \\
\text { En este mismo ámbito el entrevistado } 1 \text { (A1) menciona como } \\
\text { importante la consideración del factor empleabilidad, como } \\
\text { condicionante del desarrollo de la capacidad emprendedora como } \\
\text { parte de la formación de los ingenieros. } \\
\text { Los supervisores consideran que las habilidades puestas en práctica } \\
\text { por los estudiantes son pertinentes al contexto profesional, entre ellas } \\
\text { destacan la capacidad para solucionar problemas y la orientación } \\
\text { al cumplimiento de tareas y metas asignadas. }\end{array}$ & $\begin{array}{l}\text { "primero, debe dimensionar el problema y determinar } \\
\text { cuál es el problema, condiciones que están en torno } \\
\text { a ese problema. Qué herramientas se necesitan para } \\
\text { resolverlo, esto significa estudio constante y aprendizaje } \\
\text { constante" (A1). } \\
\text { “....aparte, que el liderazgo en lo que son las capacidades } \\
\text { profesionales se ha mencionado bastante en los } \\
\text { supervisores, la capacidad de liderazgo" (A3). } \\
\text { “...aquellas actitudes y conocimiento que tiene el } \\
\text { estudiante (respecto a las competencias genéricas), que } \\
\text { lo hacen parte de una sociedad académica, profesional } \\
\text { e industrial" (A2). } \\
\text { "....uno ve una similitud de las competencias ABET } \\
\text { con las de la Universidad. Pienso que, si eso ya está a } \\
\text { nivel institucional, y es común y aceptado forma parte } \\
\text { del proceso de acreditación..." (A3). } \\
\text { "Ahora, con el mundo globalizado esas competencias } \\
\text { disciplinares tienen que ver con el trabajo colabo- } \\
\text { rativo, la capacidad de comunicación, tener un } \\
\text { segundo idioma; que en un momento se habló de un } \\
\text { tercer idioma como francés" (A3). } \\
\text { “...ya que en el mundo que les toca vivir, los empleos } \\
\text { se están enfriando y no creando" (A1). } \\
\text { "....donde muchos de ellos van a tener que crear } \\
\text { empleos, el primero es el empleo propio" (A1). } \\
\text { “...tener al menos la experiencia, con gente que ha } \\
\text { tenido empresas, que han sido exitosos; y que han } \\
\text { fracasado" (A1). }\end{array}$ & $\begin{array}{l}\text { "Se integra bien a los equipos de } \\
\text { trabajo, propone soluciones a las } \\
\text { dificultades" ( } 10 \text { y S12). } \\
\text { "Destaca la capacidad de sinte- } \\
\text { tizar de buena manera los compro- } \\
\text { misos" (S2). }\end{array}$ \\
\hline Nuevas competencias genéricas & Evidencia docente & Evidencia empresa \\
\hline $\begin{array}{l}\text { Los supervisores destacan muchas competencias genéricas que no } \\
\text { están declaradas en el modelo universitario y que son importantes } \\
\text { para la formación de un ingeniero. En los informes de práctica } \\
\text { algunos de ellos relevan distintos aspectos entre ellos el normativo, } \\
\text { puntualidad, respeto, responsabilidad y colaboración. } \\
\text { Por otra parte, se destaca la proactividad y el compromiso como } \\
\text { una actitud fundamental, como se devela en el siguiente comentario } \\
\text { Otro aspecto importante es la seguridad y confianza en sí mismo, } \\
\text { los supervisores resaltan cuando esta cualidad se presenta y por el } \\
\text { contrario cuando aún debe trabajarse } \\
\text { En el relato de los supervisores se encuentra que relevan la } \\
\text { competencia autogestión. } \\
\text { En este ámbito se mencionan dos aspectos principalmente, por una } \\
\text { parte: la competencia que destacan y la que consideran en déficit. } \\
\text { Respecto del primer punto, la competencia genérica más destacada } \\
\text { por lo supervisores en el apartado comentarios del informe escrito } \\
\text { de práctica, corresponde a disposición para el aprendizaje. } \\
\text { Respecto del aspecto deficitario los supervisores destacan, que las } \\
\text { habilidades comunicativas, tanto orales como escritas debieran mejorar. }\end{array}$ & & 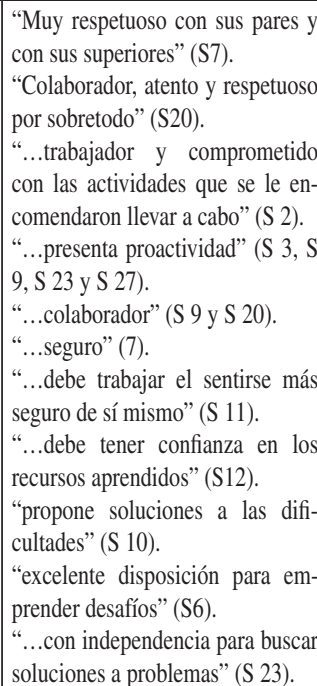 \\
\hline
\end{tabular}

Fuente: Elaboración propia.

Es importante destacar que se consideraron para este apartado categorías especiales, es decir propias del ámbito de estudio, entre ellas: Presencia-Ausencia de las competencias genéricas; Importancia de las competencias genéricas; Pertinencia de las competencias genéricas y;
Otras competencias genéricas como categoría emergente.

8 ABET: Accreditation Board for Engineering and Technology. 
Tabla 3. Matriz de análisis cualitativo sobre competencias genéricas (opinión docente).

\begin{tabular}{|c|c|}
\hline Programas de estudios & Evidencia docente \\
\hline $\begin{array}{l}\text { Se reconoce la presencia de la capacidad de autoaprendizaje y } \\
\text { comunicación oral y escrita en los programas del plan de estudios. } \\
\text { En el ámbito de la actividad curricular de práctica profesional } \\
\text { se mencio na que el aspecto de emprendimiento declarado en } \\
\text { una de las competencias no se puede desarrollar, ya que el } \\
\text { perfil de la labor del estudiante es principalmente de ejecutor. } \\
\text { Es decir, el estar a cargo condicionaría el desarrollo de la } \\
\text { capacidad de emprendimiento, es decir la competencia de } \\
\text { emprendimiento en el discurso del docente A1 se ve sólo } \\
\text { relacionada con la creación de una empresa. } \\
\text { Por otra parte, la competencia de responsabilidad social no está } \\
\text { considerada en forma explícita en el programa de la práctica } \\
\text { profesional en la carrera de Ingeniería Civil Eléctrica, lo que } \\
\text { puede explicarse a través del discurso del académico } 1 \text { (A1), } \\
\text { que menciona que el ejercicio de la responsabilidad social } \\
\text { estaría asociado solamente al ejercicio de la vida profesional. } \\
\text { Sin embargo, en ese mismo ámbito el entrevistado (A3) advierte } \\
\text { que este aspecto, es trabajado aparte de las formaciones integrales, } \\
\text { en formaciones extracurriculares, y que son convalidadas luego } \\
\text { como formaciones integrales. }\end{array}$ & $\begin{array}{l}\text { "En la asignatura de Accionamientos Eléctricos estamos } \\
\text { trabajando la capacidad del autoaprendizaje y comunicación } \\
\text { efectiva" (A1). } \\
\text { "la capacidad emprendedora no se ve en la práctica, porque es } \\
\text { una práctica, es un trabajo que por definición se ejecuta bajo } \\
\text { la mirada de un tercero. Por lo tanto, no tienes como ver esa } \\
\text { competencia bajo las formas que se está haciendo la práctica. } \\
\text { No tienes como ver este tipo de competencia, a no ser que les } \\
\text { pasaras como práctica tener su propia empresa" (A1). } \\
\text { "Pero responsabilidad social..., depende pues, ellos no están } \\
\text { ahí para proponer" (A1). } \\
\text { “... también hubo un momento en que el tema del censo, la } \\
\text { participación de los estudiantes como censista, fue convalidada } \\
\text { como formación integral" (A3). }\end{array}$ \\
\hline Metodología de enseñanza-aprendizaje & Evidencia docente \\
\hline $\begin{array}{l}\text { Los docentes, dan cuenta del desarrollo explícito de la } \\
\text { competencia genérica en alguna situación de aprendizaje. } \\
\text { Es más, el (A3), considera que el recurso metodológico } \\
\text { constituiría una oportunidad para trabajar la competencia } \\
\text { genérica de manera transversal. Dentro de las competencias } \\
\text { genéricas que mencionan son abordadas bajo esta lógica } \\
\text { encontramos: la comunicación oral y escrita, el liderazgo y } \\
\text { el trabajo colaborativo. }\end{array}$ & $\begin{array}{l}\text { “...hay dos módulos de diseño donde tiene que comunicar la } \\
\text { información de manera escrita y oral" (A1). } \\
\text { “... para el trabajo colaborativo, por ejemplo, cuando se trabaja } \\
\text { en grupo, no es fácil. Cada uno tiene sus potencialidades, } \\
\text { pero es absolutamente necesario la integración de un grupo } \\
\text { de pares" (A2). } \\
\text { “... tiene que hacer proyectos, yo les evalúo la manera de } \\
\text { expresar la redacción, después ellos tienen que hacer una } \\
\text { presentación oral, también le evalúo todo lo que tenga que ver } \\
\text { con la presentación oral, y más o menos, trato de ver el trabajo } \\
\text { colaborativo, la capacidad de comunicación" (A3). } \\
\text { "En docencia de laboratorio hago que jueguen roles de } \\
\text { comportamiento y les asigno tareas de jefatura, supervisión. } \\
\text { Estas tareas tienen que ver con el desempeño que hacen en el } \\
\text { laboratorio, y en el laboratorio nos interesa mucho la seguridad } \\
\text { personal. Y es una actitud personal, el supervisor generalmente } \\
\text { piensa que es él quien hace las cosas, pero él vela que el resto } \\
\text { cumpla algunos deberes" (A2). }\end{array}$ \\
\hline Evaluación de la competencia genérica & Evidencia docente \\
\hline $\begin{array}{l}\text { Los académicos reconocen instancias de evaluación de alguna } \\
\text { de las competencias genéricas. La actividad curricular de } \\
\text { práctica se evalúa en un protocolo de informe de práctica, } \\
\text { constituyéndose la práctica en una instancia formal de evaluación } \\
\text { de competencias genéricas. } \\
\text { Otro elemento interesante, es que parte de la evaluación de } \\
\text { práctica se realiza de manera tripartita, con el docente de la } \\
\text { Universidad, el estudiante y el supervisor de la industria. } \\
\text { Observándose una tendencia hacia la democratización del } \\
\text { proceso evaluativo de la práctica. } \\
\text { Existen criterios distintos para evaluar una misma actividad } \\
\text { curricular, ya que el tratamiento de las competencias genéricas } \\
\text { depende de la subjetividad de los/las docentes. } \\
\text { La práctica profesional contempla instrumentos formales que } \\
\text { incluyen poner en ejecución las competencias genéricas. Las } \\
\text { competencias son valoradas en base a una calificación siendo } \\
\text { bien valoradas por la empresa. } \\
\text { Como resultado de la evaluación, se observa debilidad en la } \\
\text { competencia asociada a comunicación (A1) y, liderazgo y } \\
\text { trabajo en equipo (A3). } \\
\text { El docente (A3) menciona la necesidad de incorporar una } \\
\text { evaluación más intencionada de las competencias genéricas. }\end{array}$ & $\begin{array}{l}\text { "A veces hay actividades de práctica que son muy interesantes } \\
\text { porque entonces tienen que mejorar aún más la forma en que } \\
\text { entregan la información de lo que hicieron, uno ve que la práctica } \\
\text { fue muy buena, pero el informe estuvo ahî" (A1). } \\
\text { "El protocolo donde ahí se evidencian las competencias porque } \\
\text { el protocolo me dirá como está avanzando el estudiante o } \\
\text { cumpliendo" (A2). } \\
\text { "Si he visto que en algunos dice que le falta más liderazgo. } \\
\text { También la parte de trabajo colaborativo, esto he visto en } \\
\text { los informes que me ha tocado revisar a mí; como no muy } \\
\text { desarrollada en el alumno en particular" (A3). }\end{array}$ \\
\hline
\end{tabular}




\begin{tabular}{|c|c|}
\hline Proceso reflexivo: Una categoría emergente & Evidencia Docente \\
\hline $\begin{array}{l}\text { El docente } 3 \text { (A3) hace énfasis en los siguientes aspectos: } \\
\text { a. necesidad de mejora, b. necesidad de implementación } \\
\text { de un proyecto para la mejora y c. necesidad de incluir a la } \\
\text { comunidad académica en la propuesta de mejora. A partir de } \\
\text { ello el docente, durante la entrevista, reflexiona sobre lo hasta } \\
\text { ahora implementado, poniendo un acento en la mejora. Así } \\
\text { también en desarrollar estrategias concretas para avanzar en lo } \\
\text { que considera deficitario, dentro de lo que se destaca la falta de } \\
\text { capacitación docente para trabajar las competencias genéricas. } \\
\text { También, desde el discurso se desprende, que la participación } \\
\text { de los/las académicos/as en la definición del plan operativo, } \\
\text { se consideraría como fundamental. } \\
\text { Por otra parte, resulta interesante, ver como el docente A3 } \\
\text { durante la entrevista logra visualizar como debilidad el } \\
\text { tratamiento que se le ha dado a las competencias genéricas al } \\
\text { interior de la carrera. Lo que en un inicio se planteaba como } \\
\text { normal, luego se modifica y visualiza por el mismo docente, } \\
\text { como una posibilidad de mejora. }\end{array}$ & $\begin{array}{l}\text { "... Pero lo que se ha pensado o he estado pensando es incentivar } \\
\text { a los profesores que hagan actividades. Por ejemplo, que } \\
\text { sean más rigurosos en la entrega de los informes, lo que es la } \\
\text { redacción. Justificar, argumentar lo que ellos escriben, que sean } \\
\text { más rigurosos en cómo sean los trabajos" (A3). } \\
\text { "...tendría que conversar con los profesores..., y hacer un } \\
\text { plan de trabajo común donde estemos de acuerdo hacia dónde } \\
\text { llevaremos esas evaluaciones para incentivar donde tenemos } \\
\text { la falencia" (A3). } \\
\text { Respecto a las competencias genéricas el docente al inicio de } \\
\text { la entrevista menciono que “... pero si bien está declarado en } \\
\text { el programa, yo no lo visualizo como lo más importante dentro } \\
\text { del programa" (A3). } \\
\text { "...yo diría que permite que el alumno tenga esas competencias, } \\
\text { pero para mejorarlas, sería bueno que se le diera más importancia } \\
\text { dentro de la especialidad” (A3). }\end{array}$ \\
\hline
\end{tabular}

Fuente: Elaboración propia.

El análisis que se muestra a continuación es propio de categorías asociadas a los discursos de los docentes evaluadores de las prácticas profesionales.

\section{DISCUSIÓN ENTRE EL DISCURSO ACADÉMICO Y EL DE LA INDUSTRIA}

A continuación, se darán a conocer los principales aspectos que permiten vincular los discursos académicos y de la industria, respecto a las competencias genéricas, a partir de lo evidenciado en los apartados anteriores.

Como primer elemento, se debe señalar que la valoración de la empresa mayoritariamente es consistente, considerando tanto la apreciación numérica como el discurso narrativo. Lo que se evidencia, por ejemplo, en que ambos muestran valoraciones equivalentes para las distintas competencias. Así por ejemplo, la competencia: Capacidad para comunicarse se etiqueta baja en cuanto a puntuación, lo que se corrobora con la apreciación del discurso narrativo donde también se le considera como un factor a mejorar en los/las estudiantes. Lo mismo ocurre con la competencia Disposición para el aprendizaje, siendo aquí el fenómeno a la inversa, ya que en ambos apartados es suficientemente bien valorada.

Respecto de la evaluación de la competencia Capacidad emprendedora y de liderazgo, se evidencia una puntuación también baja. Sin embargo, el discurso textual no es explícito para ratificar este punto, pudiendo advertir cierta relación con la mención de aspectos tales como; proactividad y autogestión, sobre las que sí se pronuncia en el apartado Otras competencias genéricas. En cuanto a la competencia Trabajo colaborativo, este es bien valorado en el apartado numérico, más no hay un mayor pronunciamiento en el apartado observaciones. Lo que podría explicarse justamente por la alta valoración numérica.

Ahora bien, respecto a la distinción o contrastación entre el discurso académico y de la industria, se puede decir que son coincidentes en señalar que la competencia genérica: Capacidad para comunicarse, debe fortalecerse en los estudiantes, considerando que ambos mencionan que esta es una de las competencias fundamentales para el ejercicio de la profesión.

Por otra parte, también existe coincidencia en la apreciación de la debilidad de la competencia Emprendimiento y liderazgo, difiriendo en este caso, en la interpretación; lo que se refleja en que la academia posee algunas pre-concepciones respecto de lo que se entendería por emprendimiento; las que se alejarían del discurso institucional en cuanto a su descripción. Mientras que la industria en las observaciones, realiza aseveraciones que podrían asociarse a un buen desempeño en este ámbito, tales como; resolución de problemas, iniciativa, cumplimiento de objetivos. Lo que hace ver, que existiría también una diferenciación en cuanto a la definición conceptual de esta competencia. 
Cabe destacar, que la industria valora otros aspectos a destacar que no están considerados dentro de las competencias genéricas actuales declaradas por la Universidad del Bío-Bío, tales como; algunos aspectos normativos, el respeto y la responsabilidad en el entorno laboral, y la colaboración. También se enfatiza, la proactividad, el compromiso y la autogestión, la seguridad y la confianza en sí mismo.

Así también, es importante señalar que existe diferencia respecto de la importancia otorgada por los/las académicos/as y la industria al desarrollo de las competencias genéricas. Mientras que los primeros reconocen en ella una importancia relativa, la industria pondría mayor énfasis en este aspecto, lo que es posible visualizar sobre todo en el apartado observaciones, donde mayoritariamente hacen alusión a este tipo de competencia para valorar el desempeño de los/las estudiantes en práctica profesional.

Lo anterior, pone de manifiesto lo ya expresado por diversos autores respecto de la falta de acuerdo del significado de las competencias [17, 18], lo que advierte la necesidad de una mayor vinculación entre la academia y la industria para abordar estos temas. Por otra parte, la importancia otorgada por los académicos, abre el debate respecto del trabajo de las mismas en la formación profesional, evidenciando la necesidad de fortalecer las estrategias para incluirlas en el desarrollo curricular de manera explícita [3, 19-23] tal como lo plantean los distintos referentes internacionales [1-3, 5-6, $11,15-16,18,25-28]$.

En ese mismo contexto, cobra relevancia el sentido de la práctica profesional como el escenario idóneo para la evaluación de las competencias, lo que implica una mayor vinculación entre la academia y la industria para acordar marcos de referencia comunes para medir desempeños [30-31], entendiendo que el contar con un referente externo es de relevancia para lograr una mejor comprensión de las implicancias del proceso formativo.

Por último, coincidiendo con Bolívar [36, 37], al ser la evaluación de competencias uno de los aspectos más complejos, se requiere avanzar hacia una evaluación colegiada que permita objetivar de mejor manera el proceso formativo bajo el modelo institucional y los referentes de la ingeniería a nivel Global, por ejemplo, como lo evidencian las propuestas vinculación Universidad - Industria [11].

\section{CONCLUSIONES}

Respecto al objetivo específico uno, referido a identificar en el discurso académico la presencia de las competencias genéricas declaradas por la universidad en el proceso de práctica profesional de la carrera de Ingeniería Civil Eléctrica, se puede decir que los docentes recuerdan con ciertas imprecisiones las competencias genéricas declaradas en la universidad, conceptualizándolas acorde a esquemas propios que en ocasiones difieren de la declaración prescriptiva. No obstante, al revisarlas los tres académicos, le otorgan importancia a este aspecto en la formación de ingenieros no teniendo resuelto al parecer, el cómo desarrollarlo, lo que provoca distintas miradas respecto a esta idea.

Así también para el objetivo específico dos, que decía relación con describir desde la visión del supervisor de práctica de la empresa el desempeño basado en competencias genéricas de los estudiantes en práctica profesional de la carrera de Ingeniería Civil Eléctrica. Se puede afirmar que, la visión del supervisor es positiva respecto a las competencias genéricas de los/las estudiantes, ya que las cuatro C.G. declaradas por el programa tienen un promedio de 6,62 en una escala de 1 a 7 , existiendo una mínima variación entre la competencia de capacidad emprendedora y liderazgo y la capacidad para comunicar ideas y/o sentimientos.

En relación a la contrastación, de los discursos académicos e industrial, las visiones del mundo académico y de la empresa respecto a las competencias genéricas en la carrera de Ingeniería Civil Eléctrica, se puede decir que existe relativa convergencia entre el discurso de la empresa (numérico y opinión) y el académico respecto a la importancia que le asignan a las competencias genéricas en la formación de ingenieros. Pudiendo ser esta mayormente reflejada en el discurso de la industria. En ese mismo sentido, ambos discursos son coincidentes en el énfasis que le asignan a la competencia genérica capacidad para comunicarse, y en la necesidad de reforzar la misma en el itinerario formativo. Por otra parte, se observa diferenciación cuanto a la compresión respecto al significado de cada competencia genérica. 
Es interesante relevar que todos los supervisores de práctica cuando hacen sus comentarios mayoritariamente los hacen sobre las competencias genéricas, no se refieren a las competencias específicas en sí mismas, sino vinculadas a un desarrollo integral de un desempeño. Cabe destacar, que se observaron ciertas imprecisiones en la forma de conceptualizar las competencias genéricas, principalmente al momento de preguntar por ellas a los académicos, y solicitar la opinión sobre el desempeño a los supervisores. Dicho sesgo, estaría en la falta de familiaridad de ambos actores con indicadores de desempeño que permitiesen valorar más objetivamente cada competencia. Por lo que se sugiere, mejorar el diseño y construcción del instrumento para evaluar en base a lo anterior.

Un aspecto que llama la atención, es la ausencia de la competencia genérica responsabilidad social. Si bien, la cual no tributaría al programa de Práctica Profesional en la carrera de Ingeniería Civil Eléctrica; dicha competencia forma parte de las institucionales, y el escenario de práctica constituye una buena instancia para medir aquello. Cabe destacar, que aun cuando este aspecto está considerado en los referentes internacionales; ni la academia, ni la industria mencionan esta competencia en el proceso de práctica.

Finalmente, se reconoce como importante la reflexión desarrollada por uno de los entrevistados, quien a partir de este proceso indagativo advierte la necesidad de revisar el tratamiento de las competencias genéricas al interior de la carrera.

Así también, tal como señalan los autores de referencia, la evaluación de las competencias debe considerar criterios de calidad de conocimiento público, los que deberán ser socializados a los/las estudiantes, equipo docente y sector industrial, avanzando a generar un contexto de conocimiento reflexivo, el que por medio del uso de instrumentos objetivos permita avanzar en el conocimiento del grado de avance de las metas a alcanzar y el rol de los distintos actores para ayudar a su cumplimiento.

\section{AGRADECIMIENTOS}

Los autores agradecen a la Universidad del BíoBío, por el apoyo prestado a esta publicación, a través del proyecto de Investigación e Innovación en Docencia.: "Evaluación dual entre la industria y la academia para la valoración del desempeño de estudiantes en Práctica Profesional de las carreras de Ingeniería Civil con planes armonizados", Código: 1814143/IDU.

\section{REFERENCIAS}

[1] R. Alsina, et al. "Evaluación por competencias en la universidad: las competencias transversales". Biblioteca Digital de la Universidad de Barcelona IDP-ICE (Institut de Desenvolupament Professional-ICE) A - Llibres Universitat (IDP-ICE, Octaedro), pp. 64. Páginas consultadas 26-28. Febrero2011. ISSN, en línea. DOI: http://hdl.handle. net $/ 2445 / 145000$

[2] A. Díaz. "Armonización curricular en la Educación Superior". Revista Electrónica Educare 10.1, pp. 25. Páginas consultadas 3-28. 2007. Fecha de la consulta 10/05/2018. URL: http://www.redalyc.org/articulo. oa?id=194119261002.

[3] A. Villa y M. Poblete. "Evaluación de competencias genéricas: principios, oportunidades y limitaciones". Bordón, Revista de pedagogía. Vol. $63 \mathrm{~N}^{\circ} 1$, pp. 24. Páginas consultadas: 147-170. Febrero, 2011. ISSN: 0210-5934. DOI: Dialnet-Evaluacion De Competencias Genericas-3601062.pdf

[4] V. Guichot. “¿Qué universidad estamos construyendo? Pasado y presente de algunas concepciones sobre la universidad". Cuestiones pedagógicas, 19, pp. 32. Páginas consultadas: 11-39, 2008. Fecha de la consulta 15/05/2018. URL: https://idus.us.es/bitstream/ handle/11441/12830/file_1.pdf;jsessionid= 8E212676B887889CA7886A55D9319E74 ?sequence $=1$

[5] J. González, R. Wagenaar y P. Beneitone. "Tuning-América Latina: un proyecto de las universidades". Revista iberoamericana de educación. Vol. 35 No 1, pp. 14. Páginas consultadas: 151-164. Mayo - Agosto, 2004. ISSN: 1681-5653 (en línea). DOI: https://www. researchgate.net/profile/Robert_Wagenaar2/ publication/28078676_Tuning-America_ Latina_un_proyecto_de_las_universidades/ links/0deec53c579b1998b4000000/ 
Tuning-America-Latina-un-proyecto-delas-universidades.pdf

[6] P. Beneitone y C. Esquetini. "Reflexiones y perspectivas de la Educación Superior en América Latina (Informe Final. Proyecto Tuning. América Latina 2004-2007)". Bilbao: Publicaciones de la Universidad de Deusto, pp. 378-429. ISBN: 978-84-983007-8-9. 2007.

[7] R. Pey y S. Chauriye S. "Innovación curricular en las universidades del consejo de rectores 2000-2010". Consejo de Rectores de Universidades chilenas (CRUCH), pp. 91. Páginas consultadas: 65-67; 2011. Fecha de la consulta 17/05/2018. URL: http://vra. ucv.cl/ddcyf/wp-content/uploads/2013/06/ Informe_innovacion_curricular.pdf

[8] J. Delors. "La educación encierra un tesoro". Santillana-UNESCO. Vol. 1, capítulo 4, pp. 13. Páginas consultadas: 91-103. Madrid, España. ISBN:968-7474-00-9. 1996.

[9] A. Rodríguez. "Las competencias en el Espacio Europeo de Educación Superior: tipologías". Humanismo y Trabajo Social, 6, pp. 139-153. Marzo, 2007. ISSN: 1696-7623.

[10] K. Wall. "Engineering: Issues, Challenges and Opportunities for development". UNESCO Report. Primera Edición, pp. 1-394. Páginas consultadas: 39-50. Paris, Francia. ISBN: 978-923-104156-3. 2010.

[11] F. Falcone, et al. "Implementation of Higher Education and Life Long Learning Curricula based on University-Industry Synergic Approach". International Journal of Engineering Education, Vol. 35, $\mathrm{N}^{\mathrm{o}} 6$, pp. 1568-1583. Páginas consultadas 15721581; 2019. Fecha de la consulta 03/03/2020.

[12] G. Le Boterf. "L'ingénierie des competénces". Colección Formación y desarrollo. Edición $1^{a}$ ed., pp. 1-461. Barcelona. España. 2001. ISBN: 84-8088-529-7

[13] S. Whiddett and S. Hollyforde. "A practical guide to competencies: how to enhance individual and organisational performance". CIPD Publishing, 2n Edition, pp. 138. Páginas consultadas: 24-50. London, England. ISBN: 1-84398-012-6. 2003. URL: https://books. google.es/books?hl=es\&lr=\&id=ZP850cgpG I0C\&oi=fnd\&pg=PP11\&dq=Whiddett, + S., $+\% 26+$ Hollyforde, + S.+(2003) + A+practic al+guide+to+competencies:+how+to+enha nce+individual+and+organisational+perfor mance. + CIPD+Publishing.\&ots $=$ Md $92 \mathrm{VxQ}$ $6 \mathrm{aM} \&$ sig $=$ WQFvOnamk0Ltu7k1JkLaPnk $\mathrm{YWn} 0 \# \mathrm{v}=$ onepage $\& \mathrm{q}=$ Whiddett $\% 2 \mathrm{C} \% 20$ S. $\% 2 \mathrm{C} \% 20 \% 26 \% 20 \mathrm{Hollyforde} \% 2 \mathrm{C} \% 20$ S.\%20(2003).\%20A\%20practical\%20 guide $\% 20$ to $\% 20$ competencies $\% 3 \mathrm{~A} \% 20$ how $\% 20$ to $\% 20$ enhance $\% 20$ individual $\% 20$ and $\% 20$ organisational $\% 20$ performance. $\% 20$ CIPD\%20Publishing. \&f $=$ false

[14] R.K.G. Marx and S.M.G. Puente. "La Evaluación con Enfoque por Competencias: ¿se implementa realmente la evaluación por competencias?". Revista Electrónica de Desarrollo de Competencias, Universidad de Talca. Vol. $1 \mathrm{~N}^{\circ} 3$, pp. 22. Páginas consultadas 104-125. Talca, Chile. 2009. URL: http:// dta.utalca.cl/ojs2/index.php/fcompetencias/ article/view/41/44

[15] A. Villa, M. Poblete y otros. "Aprendizaje basado en competencias. Una propuesta para la evaluación de las competencias genéricas". Mensajero. pp. 336. Bilbao. España. 2007. ISBN: 978842712833-0

[16] M. Orsini. "Temas transversales: una experiencia de aula desde la educomunicación", Revista Científica de Comunicación y Educación, $N^{\circ} 24$, pp. 94104. Páginas consultadas 99-104. Marzo, 2005. ISSN: 11343478.

[17] J. Carreras., \& P. Perrenound. "El Debat sobre les competències a l'ensenyament universitari”. ICE Universitat de Barcelona, pp. 54. Páginas consultadas 8-13. Barcelona, España. ISBN: 84-88795-87-4. 2005.

[18] M. Van der Klink., J. Boon., \& K. Shlusmans. Competencias y formación profesional superior: presente y futuro. Revista Europea de formación profesional, $\mathrm{N}^{\circ}$ 40, Páginas consultadas 74-91. Enero, 2007. ISSN (en línea): 1977-0235.

[19] J. Gimeno Sacristán, et al., "Educar por competencias, ¿Qué hay de nuevo? "Editorial Morata, S.L. $3^{\circ}$ ed. pp. 233. Páginas consultadas 225-231. Madrid, España. ISBN: 978-84-7112-528-6. 2008.

[20] C. Font., et al. "Pisa como excusa: Repensar la evaluación para cambiar la enseñanza". Editorial GRAÓ, $1^{\circ}$ edición, pp. 191. Páginas consultadas 98-100. Barcelona, España. ISBN: 978-84-7827-706-3. 2009. 
[21] L. Cazares. "Estrategias educativas docentes para fomentar competencias. Crearlas, organizarlas, diseñarlas y evaluarlas (CODE)". Trillas. $1^{\circ}$ edición, 1-184. México. 2011. ISBN 10: 6071708230

[22] L. Villardón-Gallego (coord.) "Competencias genéricas en Educación Superior. Metodologías específicas para su desarrollo". Narcea, S.A de Ediciones. Vol. $27 \mathrm{~N}^{\mathrm{o}} 1$, pp. 190. Páginas consultadas 13-24. Madrid, España. ISBN (impreso): 978-84-277-2077-0. ISBN (en línea): 978-84-277-2078-7. 2015.

[23] M. Poblete., et al. "La formación del docente en competencias genéricas: un instrumento para su planificación y desarrollo". Revista Educar, vol. 52/1, pp. 21. Páginas consultadas 71-91. Septiembre, 2015. ISSN (en línea): 2014.8801. ISSN (impreso): 0211-819X. DOI: https://doi.org/10.5565/rev/educar.713

[24] M.D. Villa. "Del campo curricular en América Latina: elementos para su comprensión". [Con] textos. Vol. $5 \mathrm{~N}^{\mathrm{o}} 20$, pp. 12. Páginas consultadas 23-34. December, 2016. DOI: 10.21774/ctx.v5i20.755

[25] M. Harrison. "Jobs and Growth: The Importance of Engineering Skills to the UK Economy". Royal Academy of Engineering Econometrics of Engineering Skills Project. Final Report, September 2012.

[26] E. Dowell, E. Baum and J. Mctague. "The Green Report: Engineering education for a changing world. American Society of Engineering Education", pp. 84. Páginas consultadas: 3-6. 1995. 25/09/2018. URL: https://apps.dtic.mil/dtic/tr/fulltext/u2/ a293931.pdf

[27] R.M. Felder and R. Brent. "Designing and teaching courses to satisfy the ABET engineering criteria". Editorial: Journal of Engineering Education. Vol. 92 N $^{\circ}$ 1, pp. 7-25. Enero, 2013. URL: https://onlinelibrary.wiley. com/doi/epdf/10.1002/j.2168-9830.2003. tb00734.x

[28] K. Wall. "Engineering: Issues, challenges and opportunities for development". UNESCO, pp. 5. Páginas consultadas: 299-303. Paris, Francia. ISBN: 978-92-3-104156-3. 2010.

[29] A. Patil. "The global engineering criteria for the development of a global engineering profession". World Transaction on Engineering Education. Vol. $4 \mathrm{~N}^{\mathrm{o}}$ 1, pp. 49-52. Páginas consultadas: 50.
Melboune, Australia. 2005. URL: https://pdfs. semanticscholar.org/2287/0adcc71aebc5e8ed 083b0f00b48a5f06d171.pdf

[30] A. Eizagirre., et al. "Las competencias transversales en el grado de Pedagogía: diagnóstico y estado de la cuestión". REDU. Revista de Docencia Universitaria. Vol. 15 $\mathrm{N}^{\circ} 1$, pp. 18. Páginas consultadas: 259-276. Enero- junio, 2017. ISSN: 1887-4592.

[31] A. Medina, et al. "Evaluación de las competencias genéricas y profesionales de los estudiantes". Innovación educativa (México, DF). Vol. 12 No 58, pp. 18. Páginas consultadas: 133-150. Enero-abril, 2012. ISSN: 1665-2673 DOI: http://www.scielo. org.mx/pdf/ie/v12n58/v12n58a8.pdf

[32] S. Culver, J. Hirt, E. Creamer, N. Bodenhorn and P. Burge. "Clarifyng what we do trough student out-comes assessment". European Educational Research. ASSN. August, Helsinki. 2010.

[33] G. Le Boterf. Repenser la compétence: pour dépasser les idées reçues: quinze propositions. Editions Eyrolles. $2^{\circ}$ Edición, pp. 101-103. Paris. Francia. ISBN: 978-2212-54727-6. 2010.

[34] M. Sabariego. "La evaluación de competencias transversales a través de rúbricas”. @ tic. revista d'innovació educativa. $\mathrm{N}^{\circ} 14$, pp. 9. Páginas consultadas 50-58. Enero- junio, 2015. ISSN: 1989-3477. DOI: 10.7203. 2015.

[35] MA. Zabalza. "Evaluación de actitudes y valores". En A. Medina, J. Cardona, S. Castillo y M.C. Domínguez (eds.): Evaluación de los procesos y resultados del aprendizaje de los estudiantes. Madrid, UNED, pp. 56. Páginas consultas: 245-300. 1998. Fecha de la consulta: 9/10/2018.

[36] A. Bolívar. "Non scholae sed vitae discimus: Límites y problemas de la transversalidad". Revista de Educación. $\mathrm{N}^{\circ}$ 309, pp. 23-65. Páginas consultadas 43. Enero-abril, 1996a. ISSN: 0034-8082 URL: https://www.researchgate.net/profile/ Antonio_Bolivar/publication/292608167_ Non_scholae_sed_vitae_discimus_ Limites_y_problemas_de_la_transversalidad/ links/56afd2ac08ae8e37214d1112.pdf

[37] A. Bolívar. "La evaluación de actitudes y valores: problemas y propuestas. Castillo 
Arredondo, S. Compromisos de la evaluación Educativa", pp. 23. Páginas consultadas 91114. 2002. 4/06/2018. URL: https://www. researchgate.net/profile/Antonio_Bolivar/ publication/44547008_La_evaluacion_ de_valores_y_actitudes_Antonio_Bolivar/ links/55e0759108ae6abe6e88d61b.pdf

[38] J.I. Ruiz Olabuénaga. "Metodología de la investigación cualitativa". Vol. 15. Universidad de Deusto. Vol. 5, p. 342. Páginas consultadas: 165-210. Bilbao, España. ISBN: 978-84-9830-673-6. 2012.

[39] R. Flores. "Observando observadores: una introducción a las técnicas cualitativas de investigación social”, p. 391. Páginas consultadas: 44-48. 2014. Fecha de consulta: 7/06/2018. URL: https://books.google.es/ books?hl=es\&lr=\&id=ks8oCAAAQBAJ $\&$ oi $=$ fnd $\& p g=$ PA $18 \&$ dq $=$ Flores,+ R. $+(20$ 09).+Observando+observadores:+una+in troducci\% $\% 3 \% \mathrm{~B} 3 \mathrm{n}+\mathrm{a}+\mathrm{las}+\mathrm{t} \% \mathrm{C} 3 \% \mathrm{~A} 9 \mathrm{cni}$ cas+ cualitativas+de+investigaci $\% \mathrm{C} 3 \% \mathrm{~B}$ $3 \mathrm{n}+$ social. $\&$ ots $=$ T5Q1VpzegA $\&$ sig $=1 \mathrm{pDo}$ R4TwkrWbtbl_PZScnZMM9Jc\#v=onepa ge\&q=Flores \%2C\%20R.\%20(2009).\%20 Observando $\% 20$ observadores $\% 3 \mathrm{~A} \% 20$ una $\% 20$ introducci\%C3\%B3n $\% 20 \mathrm{a} \% 20$ las $\% 20 \mathrm{t} \% \mathrm{C} 3 \% \mathrm{~A} 9$ cnicas $\% 20$ cualitativas\%20de\%20investigaci\%C3\%B3n\%20 social. $\& \mathrm{f}=$ false 